\title{
LACTATO EM TESES DE ENDURANCE E DE VELOCIDADE
}

\author{
KISS, M. A. P. D. M.* \\ MACHIDA, J.** \\ DIRANI, I.*** \\ ZUCAS, S. M. ${ }^{* * * *}$
}

\begin{abstract}
RESUMO
O objetivo do trabalho é analisar o comportamento do lactato sanguineo em testes repetidos de velocidade, os quais procuram simular as atividades intensas e intermitentes durante jogo de voleibol. Foram examinados 10 jogadores da TELESP, antes da fase de treinamento de base e 2 meses após; os testes de laboratório seguiram os protocolos de Astrand no cicloergomêtro e de Mader em esteira. Em campo cada atleta correu 5 vezes a distância de $30 \mathrm{~m}$ a velocidade

Houve diminuição do lactato no teste de esteira com o treinamento, para o subgrupo em piores condiçōes de endurance; a diminuição da produção de lactato nos tiros ocorreu apenas a partir do $2^{\circ}$ tiro; näo houve correlaçāo entre a evolução do teste de esteira e de campo. Concluimos que existe aumento de lactato já a partir do 10 tiro $e$ que há necessdade de aplicarmos dois diferentes tipos de testes para acompanhamento da intensidade de treinamento em voleibol.
\end{abstract} máxima (5 "tiros"), com coleta de sangue entre os mesmos.

* Professor Adjunto do Departamento de Organização e Aplicação Desportiva.

* Professor Assistente do Departamento de Organização e Aplicação Desportiva.

*** Técnico de Laboratório (EEFUSP).

**** Professor Titular - Chefe do Dep. de Org. e Aplicação Desportiva. 


\section{Introdução}

No estudo de atividades complexas, como jogos, há necessidade inicial de separar os tipos de trabalhos que os compõe, para depois procurarmos fazer uma sintese dos mesmos. Assim, quando um jogador de voleibol não tem bom desempenho precisamos diagnosticar as razōes as quais podem se manifestar através da diminuição na endurance (cargas que correspondem a porcentagens baixas de consumo máximo de oxigênio), resistência (porcentagem quase máxima), na velocidade etc., isto é importante, pois devido a especificidade de treinamento, cada um desses tópicos tem características distintas, devendo ser desenvolvidas de forma harmônica, mas não simultânea, para a obtenção dos melhores resultados.

A quantidade de ácido láctico no sangue foi, até há poucos anos, uma medida direta da ausência relativa de oxigênio a nivel celular (Margaria et alii, 1969; Brooks e Fahey, 1984; Kiss, 1987; Kiss et alii, 1987); atualmente ela é conhecida como dependente do padrão de recrutamento das fibras brancas, que o produzem (mesmo na presença de oxigênio) e das fibras vermelhas do mesmo músculo, que simultâneamente o utilizam (na presença de oxigênio). Outros músculos pouco ativos no momento de determinado esforço, bem como coração e fígado tem grande participação no "clearence" de lactato (Brooks, 1987).

Apesar do complexo equilíbrio metabólico existente para níveis semelhantes de ácido láctico, em sangue arterializado, eles ainda são utilizados para determinar a intensidade das cargas de trabalho (Föhrenbach, R. et alii, 1987) de forma individual e momentânea.

Poucos são ainda os trabalhos que seguem o desenvolvimento de modalidades esportivas complexas (Ekblom, 1986) por períodos superiores a 4 semanas. Qual seria a melhoria que esportistas já treinados, podem ainda obter em relação aos niveis de ácido láctico no sangue em testes de média e curta duração? Existe relação entre os dois tipos de testes?

O objetivo deste trabalho é analisar o comportamento de niveis sanguíneos de lactato em testes repetidos de velocidade, os quais simulam melhor as atividades duranto 0 jogo, pois elas são na maioria das vezes intermitentes (Ekblom, 1986). Além disso até que ponto é discutido a evolução da variável endurance $e$ da repetição de velocidade durante 2 meses de treinamento de base.

\section{Metodologia}

Foram examinados 10 atletas jogadores de voleibol, adultos, pertencentes a equipe regional da TELESP, antes e depois de 2 meses da preparação geral; esta tinha como objetivo inicial desenvolver condições aeróbicas e posteriormente mantê-las e desenvolver características de velocidade e potência.

As variáveis morfológicas de cineantropometria foram peso e estatura. Os testes funcionais utilizados além do Astrand e Rodahl em ciclo ergômetro (1970) foram o escalonado de endurance (Mader e Hollman, 1977 atualizado por Föhrenbach et alii, 1987). Assim foram aplicadas 4 cargas de forma crescente em esteira rolante, sem atingir velocidades máximas, mas elevando a freqüência cardíaca até quase a máxima para idade e sexo. A freqüência cardíaca durante exercício e após foi registrada em eletrocardiógrafo Herolet Pochard.

Foi colhido lactato em lóbulo de orelha, hiperemiado pelo uso de pomadas Finalgon. A dosagem em 20 ul de sangue (Mader e HolImann, 1977) foi feita nas amostras referentes a cada carga na esteira.

O teste de pista foi adpatado de Liesen (1985). Foram feitos 5 tiros de $30 \mathrm{~m}$; cada tiro percorrido na velocidade máxima que o indivíduo conseguia; o sangue de lóbulo de orelha hiperemiada com Finalgon era coletado imediatamente após cada tiro e não apenas como fraconizado por Liesen (1985) após o $2^{\circ}$ e $5^{\circ}$ tiros.

Os atletas estavam no início da preparação física de base no "pré-treino" e a terminar quando dos testes denominados "pós-treino", 2 meses após.

\section{Resultados e Comentários}

As características gerais dos jogadores quanto a idade e os aspectos morfológicos e 0 consumo máximo de oxigênio encontra-se na tabela I. 


\begin{tabular}{|c|c|c|c|c|c|}
\hline $\begin{array}{l}\text { IDADE } \\
\text { (anos) }\end{array}$ & \multirow{2}{*}{ 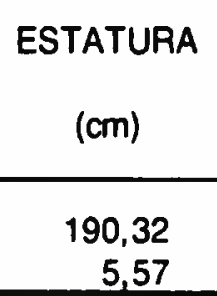 } & \multirow{2}{*}{\multicolumn{2}{|c|}{$\begin{array}{c}\text { PESO (kg) } \\
\\
\text { PÓS } \\
82,0 \\
6,80 \\
\end{array}$}} & \multicolumn{2}{|c|}{$\begin{array}{c}\mathrm{VO}_{2} \mathrm{Máx} . \\
1 / \mathrm{m}, \mathrm{ml} / \mathrm{kg}, \mathrm{mm}\end{array}$} \\
\hline $\begin{array}{cc}X & 22,3 \\
S & 2,49 \\
\end{array}$ & & & & $\begin{array}{l}3,55 \\
0,45 \\
\end{array}$ & $\begin{array}{l}4,40 \\
6,0 \\
\end{array}$ \\
\hline $\begin{array}{l}t \\
g l\end{array}$ & & & $\begin{array}{l}2,09 \\
9\end{array}$ & & \\
\hline
\end{tabular}

\section{TABELA I}

Idade, estatura, pré-treino, peso pré e pós-treino, consumo máximo de oxigênio no pré-treino, valores médios e desvio padrão para o grupo, resultado de estatística de estudantes e graus de ( $g l)$ para a evolução da variável peso.

Em decorrência das diferentes velocidades atingidas pelos jogadores na 4a carga do teste escalonado esteira, a apresentaçāo dos resultados desse teste 2 sub-grupos: GI $(n=6)$ que atingiu 14,4 e Gll $(n=4)$ que atingiu 15,6, $54 \mathrm{ml} / \mathrm{kg} / \mathrm{m}$ ou $1 \mathrm{~km}$ em 4'10" $57 \mathrm{ml} / \mathrm{kgg} / \mathrm{min}$ ou 1 em 3'50. Os resultados da $3^{a}(13,2)$ e $4^{a}$ carga $(14,4$ e 15,6), 5 serão apresentados na Tabela II House evolução significativo do grupo com sobrecarga mais baixa mostrando que uma melhoria mais intensa apenas nos atletas que não estavam tāo bem.

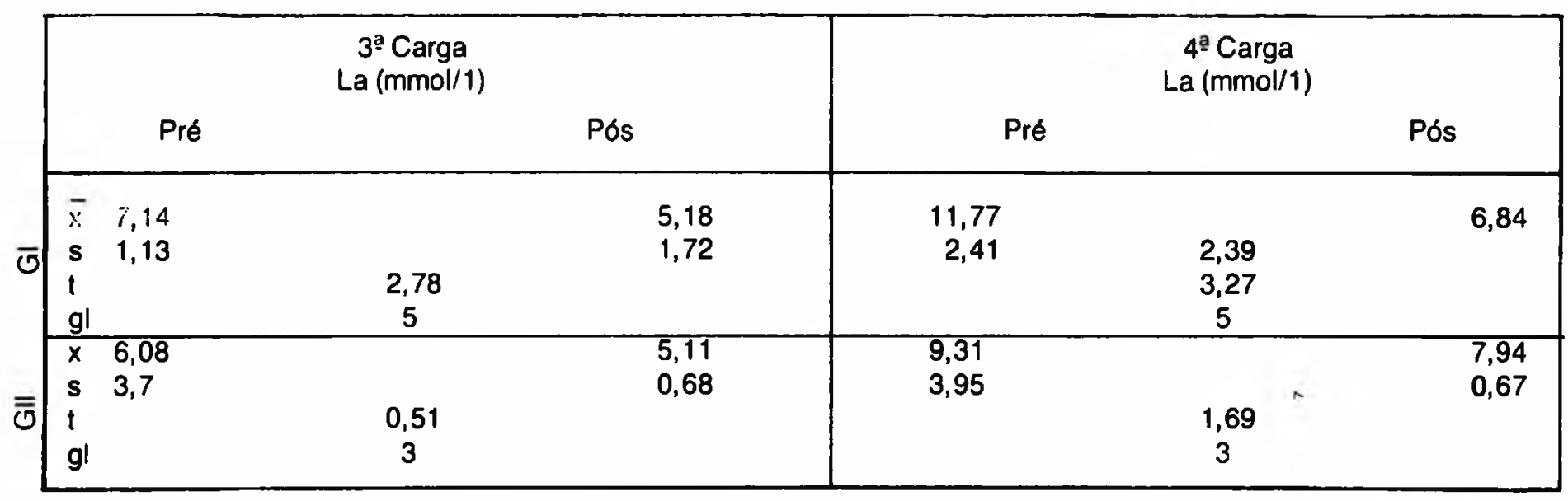

\section{TABELA II}

Lactato (mmol/1) na recuperação de cada uma das últimas duas cargas em esteira rolante em função dos grupos GI e Gll; valores médios (- $x$ desvio padrão (s), t de Student para dados correlacionados.

Os resultados dos 5 tempos de percussāo para $30 \mathrm{~m}$ e dos respectivos no pré-treino en- contram-se na Tabela III e no pós-treino na Tabela IV.

Podemos ver que já no primeiro tiro, com tempo médio de 4" e 55 já houve produção de lactato; este contudo é considerado como trabalho "alático" conceito que agora precisamos rever tempo de percussão e lactato imediatamente após cada um dos 5 tiros de $30 \mathrm{~m}$ valores médios e desvio-padrão pré-treino. 


\begin{tabular}{|c|c|c|c|c|c|c|c|c|c|}
\hline \multicolumn{2}{|c|}{19} & \multicolumn{2}{|c|}{$2^{9}$} & \multicolumn{2}{|c|}{$3^{\circ}$} & \multicolumn{2}{|c|}{$4^{\circ}$} & \multicolumn{2}{|c|}{$5^{\circ}$} \\
\hline $\bar{T}$ & La & $T$ & $\mathrm{La}$ & $\bar{T}$ & La & $\mathrm{T}$ & $\mathrm{La}$ & $T$ & $\mathrm{La}$ \\
\hline 5 & $\mathrm{mmol} / 1$ & s & $\mathrm{mmol} / 1$ & s & $\mathrm{mmol} / 1$ & $\mathbf{s}$ & $\mathrm{mmol} / 1$ & $s$ & $\mathrm{mmol} / 1$ \\
\hline $\begin{array}{l}x 4,55 \\
\text { s } 0,21\end{array}$ & $\begin{array}{l}3,03 \\
0,87\end{array}$ & $\begin{array}{l}4,52 \\
0,26\end{array}$ & $\begin{array}{l}5,17 \\
1,20\end{array}$ & $\begin{array}{l}, 44 \\
0,21\end{array}$ & $\begin{array}{l}6,48 \\
1,81\end{array}$ & $\begin{array}{l}4,53 \\
0,21\end{array}$ & $\begin{array}{l}8,17 \\
1,76\end{array}$ & $\begin{array}{l}4,42 \\
0,18\end{array}$ & $\begin{array}{l}8,42 \\
2,39\end{array}$ \\
\hline
\end{tabular}

TABELA III

Tempo de percurso e lactato imediatamente após cada um dos 5 tiros de $30 \mathrm{~m}$ : valores médios e desvio-padrão no pré-treino.

\begin{tabular}{|c|c|c|c|c|c|c|c|c|c|}
\hline \multicolumn{2}{|c|}{10} & \multicolumn{2}{|c|}{$2^{o}$} & \multicolumn{2}{|c|}{$3^{\circ}$} & \multicolumn{2}{|c|}{$4^{\circ}$} & \multicolumn{2}{|c|}{$5^{\circ}$} \\
\hline $\bar{T}$ & La & $\bar{T}$ & La & $\bar{T}$ & La & $\bar{T}$ & $\mathrm{La}$ & $\bar{T}$ & La \\
\hline $\mathbf{s}$ & $\mathrm{mmol} / 1$ & $\mathrm{~s}$ & $\mathrm{mmol} / 1$ & s & $\mathrm{mmol} / 1$ & $s$ & $\mathrm{mmol} / 1$ & s & $\mathrm{mmol} / 1$ \\
\hline$\times 4,35$ & 2,88 & 4,35 & 4,1 & 4,41 & 5,24 & 4,53 & 5,78 & 4,42 & 6,40 \\
\hline s 0,17 & 1,23 & 0,15 & 0,84 & 0,21 & 1,38 & 0,21 & 1,55 & 0,18 & 2,02 \\
\hline t 2,49 & 0,08 & $2,56^{\star}$ & $2,74^{\circ}$ & $-0,5$ & $3,47^{*}$ & $-3,33^{*}$ & $8,55^{\circ}$ & 1,13 & 2,67 \\
\hline
\end{tabular}

\section{TABELA IV}

Tempo de percurso e lactato imediatamente após cada um dos 5 tiros de $30 \mathrm{~m}$; valores médios, desvios, significância do t de Student para dados correlacionados entre pré-treino e póstreino, com 9 graus de liberdade.

Entre os 5 tiros houve diferença significativa da produção de lactato tanto no pré quanto no pós-treino (Anova para dados correlacionados $F=35,61^{\star}$ no pré e $F=15,57^{\star}$ no póstreino).

Não houve diferença significativa entre 0 delta do lactato no $5^{\circ}$ menos o do $2^{\circ}$ tiro em relação a evolução do treinamento, ou seja a diminuição foi igualmente significativa para esses tiros; este achado não confirmou o que foi

\section{4- Conclusões}

Mesmo em trabalhos conhecidos até há pouco tempo como "aláticos", ocorre aumento significativo de ácido lático, tal como ocorreu após o $1^{\circ}$ tiro em velocidade máxima; uma das causa possíveis do ácido lático não ter diminuido após treino no $1^{\circ}$ tiro poderia se o padrão encontrado por Liesen, 1985, em jogadores de futebol bem treinados, quando o treinamento de velocidade e potência fazia com que passasse a haver menor diferença entre o lactato do último e do $2^{\circ}$ tiro.

A evolução nos tempos de percurso dos tiros de $30 \mathrm{~m}$ foi irregular, conforme vemos na Tabela IV, mas por outro lado houve diminuição significativa do lactato arterializado, com exceção do logo após o $1^{\circ}$ tiro.

O coeficiente de correlação de Pearson entre o delta de evolução do lactato na quarta carga do teste escalonado de endurance na esteira e o delta de evolução no 5 o tiro do teste de velocidade foi $r=0,37$. não sendo significativo $(t=-1,139$ com nivel crítico de 1,86$)$, levando-nos a concluir pela independência das variáveis analisadas em cada teste.

inicial de recrutamento das fibras, que não teria mudado.

Interessante é verificar que provavelmente dois mecanismos distintos explicariam a diminuição do lactato com treinamento: um em provas longas e outro em provas curtas, tornando necessário dois tipos diferentes de testes para acompanhar adequadamente treinamento de voleibol. 


\begin{abstract}
The aim of this paper is to evaluate the behavior of blood lactate in repeated velocity tests, which try to simulate the intense and intermittent activity during volelyball match. Ten players of TELESP team were examined before the basic training period and two months later; laboratory tests followed Astransd's protocol on bicycle ergometer and Mader's on treadmill. On the field, each athlete run five times, 30 meters at maximal speed; blood samples were collected in treadmill

tests in subgroup worst endurance conditions. The decreased in treadmill tests in subgroup worst endurance conditions. The decrease of lactate production started after the second repeat running. There was no correlation between the evolution of the treadmill and field tests. We conclude that there is increase of lactate after the first fiel running and that is necessary to perform two different types of tests to follow the intensity of training in voleyball.
\end{abstract}

\section{REFERÊNCIAS BIBLIOGRÁFICAS}

ASTRAND, P O. \& RODAHL, K. Textbook of work physiology. New York, McGrawHill, 1970.

BROOKS, G. A. Lactate production during exercise: oxidizable substrate versus fatigue agent. In: MACLEOD, D. et alii. Exercise benefits, limits and adaptations. London. E \& F N. Spon, 1987 144-158.

BROOKS, G. \& FAHEY, T. D. Exercise physiology: human bioenergetics and its applications. New York. John Wiley \& Sons, 1984.

EKBLOM, B. Applied physiology of soccer. Sports Medicine, 3: 50-60, 1986.

KISS, M. A. P D. M. Avaliação em Educação Física: aspectos biológicos e educacionais. São Paulo, Manole, 1987
KISS, M. A. P. D. M. et alii. Limiar de lactato no teste de endurance escalonado em crianças e adolescentes. In: Congresso Brasileiro de Medicina Desportiva, 8, Rio de Janeiro, 1987. Anais ......, Rio de Janeiro, FBMD, 1987 p 6 e 20.

LIESEN, $H$. Testes de campo de futebol. Palestra proferida no Curso de Pós-Graduação em Educação Física, disciplina de Prescriçāo individualizada de treinamento. São Paulo, EEFUSP, outubro de 1985.

MADER, A. \& HOLLMANN, W. Zur bedentung der stoffwechrellersdengsfurgkeit des eliterunderers in training und wettkampf. In HECK, $H$. et alii. Ausdauertraining stoffwchsegrundlagen und ateurunwsansatze. D S B. Berlim, 1977

MARGARIA. M. et alii. Energy utilization in intermittent exercise of supramacimal intensity. J. Appl. Physiol. 26: 752-756, 1969. 